\title{
An inverted hyperplastic polyp with a characteristic colonoscopic appearance
}

A 65-year-old man was referred to our department for polypectomy. A polyp, $20 \mathrm{~mm}$ in diameter, was found in the ascending colon on colonoscopy (Figure $1 \mathbf{a}$ ). The polyp surface was covered by characteristic multiple dimples, long thin vessels, and mucus. Chromoendoscopy with $0.4 \%$ indigo carmine enhanced the appearance of the polyp surface dimples, and subsequent magnification revealed a type II pit pattern according to Kudo's criteria, leading us to a diagnosis of a large hyperplastic polyp (Figure $\mathbf{1}$ b). Most hyperplastic polyps do not show multiple dimples over their entire surface, and so an unusual histological diagnosis, such as inversion, was suspected before removal of the polyp. Endoscopic mucosal resection using an inject and cut technique was performed, and the polyp was resected en bloc. Histologically, it was indeed found to be an inverted hyperplastic polyp (Figure 2).

Inverted hyperplastic polyps of the colon were first described by Sobin et al. in 1985 [1]. They represent an unusual morphological variant of hyperplastic polyps that shows traumatic displacement of the crypt epithelium into the submucosa [1]. This polyp was characterized by multiple dimples on its surface, which could have been created by endophytic growth [2]. Hyperplastic polyps are typically small (5 mm or less in diameter), smooth, sessile lesions covered by mucus, and are located predominantly in the left colon. In contrast, hyperplastic polyps that are $10 \mathrm{~mm}$ or larger in diameter are reported to account for fewer than $1 \%$ of all hyperplastic polyps and are commonly found in the right colon. Although the polyp in our patient was diagnosed as a hyperplastic polyp on the basis of the endoscopic findings, endoscopic treatment was appropriate because large hyperplastic polyps are often reported to be associated with a component of adenoma or carcinoma, which is difficult to diagnose before removal in some cases $[3,4]$. Furthermore, four inverted hyperplastic polyps were
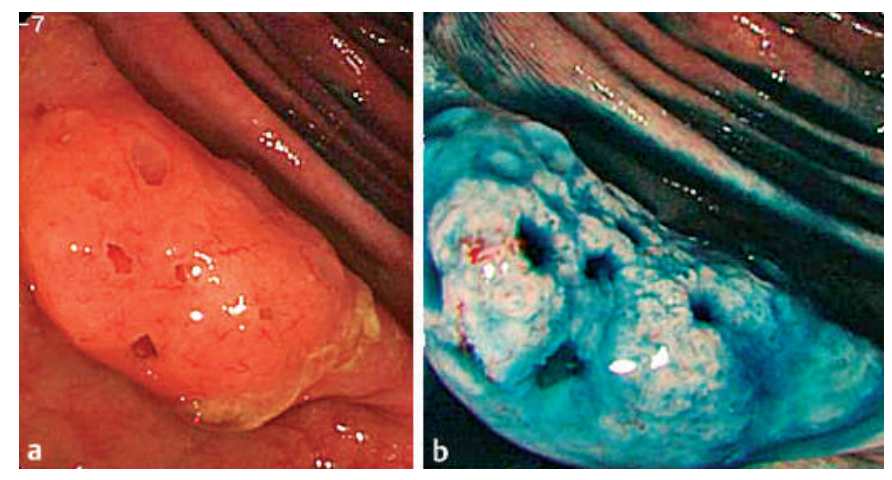

Figure 1 Colonoscopy revealed a 20mm-diameter polyp in the ascending colon; the polyp surface was covered by multiple dimples, long thin vessels, and mucus (a). Chromoendoscopy with $0.4 \%$ indigo carmine dye enhanced the characteristic dimples on the polyp's surface (b).

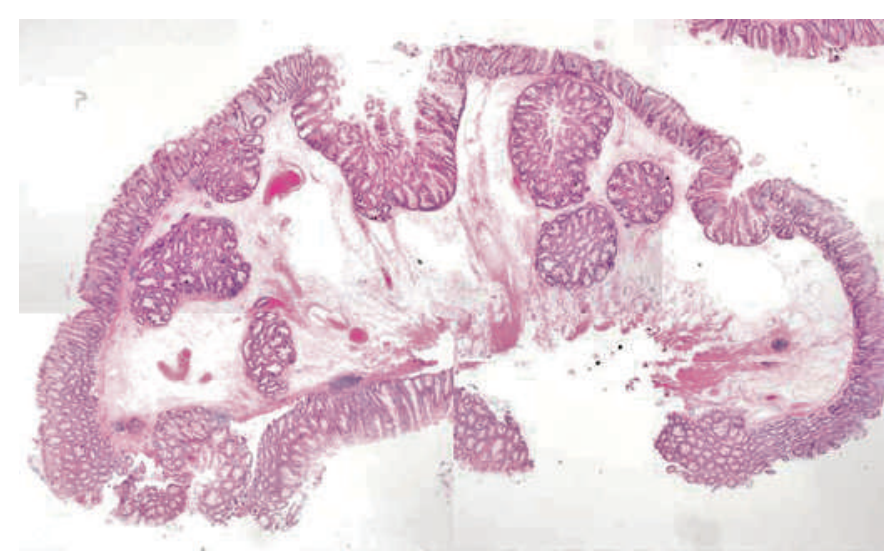

Figure 2 Histological examination showed the lesion to be a hyperplastic polyp with displaced epithelium in the submucosa.

found to be associated with adenoma in a previous report [4].

Endoscopy_UCTN_Code_CCL_1AD_2AC

K.-I. Fu'1, T. Matsuda², Y. Saito², Y. Mashimo ${ }^{2}$, S. Nonaka ${ }^{2}$, T. Fujimori ${ }^{3}$

${ }^{1}$ Department of Radiology, Dokkyo University School of Medicine, Shimotuga, Tochigi, Japan

${ }^{2}$ Gastrointestinal Endoscopy Division, National Cancer Center Hospital, Tokyo, Japan

${ }^{3}$ Department of Surgical and Molecular Pathology, Dokkyo University School of Medicine, Shimotuga, Tochigi, Japan.

\section{References}

${ }^{1}$ Sobin LH. Inverted hyperplastic polyps of the colon. Am J Surg Pathol 1985; 9: 265-272

2 Yantiss RK, Goldman H, Odze RD. Hyperplastic polyp with epithelial misplacement (inverted hyperplastic polyp): a clinicopatholo- gic and immunohistochemical study of 19 cases. Mod Pathol 2001; 14: 869-875

${ }^{3}$ Tonooka T, Sano Y, Fujii T et al. Adenocarcinoma in a solitary large hyperplastic polyp diagnosed by magnifying colonoscope: report of a case. Dis Colon Rectum 2002; 45: 1407-1411

${ }^{4}$ Kuribayashi K, Ishii T, Ishidate T et al. Two cases of inverted hyperplastic polyps of the colon and association with adenoma. Eur J Gastroenterol Hepatol 2004; 16: 107-112

\section{Corresponding Author}

\section{KI Fu, M.D.}

Department of Radiology

Dokkyo University School of Medicine 880 Kitakobayashi, Mibu Shimotuga, Tochigi 321-0293 Japan

Fax: +81-282-86-5678

E-mail: fukuangi@hotmail.com 